UCRL-JC-114264

PREPRINT

\title{
Thermo-Hydraulic Analysis of the TPX Superconducting TF Magnets
}

\author{
Robert L. Wong, Jon P. Zbasnik, William V. Hassenzahl
}

This paper was prepared for submittal to the 15th Symposium on Fusion Engineering

Hyannis, MA

October 11-15, 1993

October 6, 1993

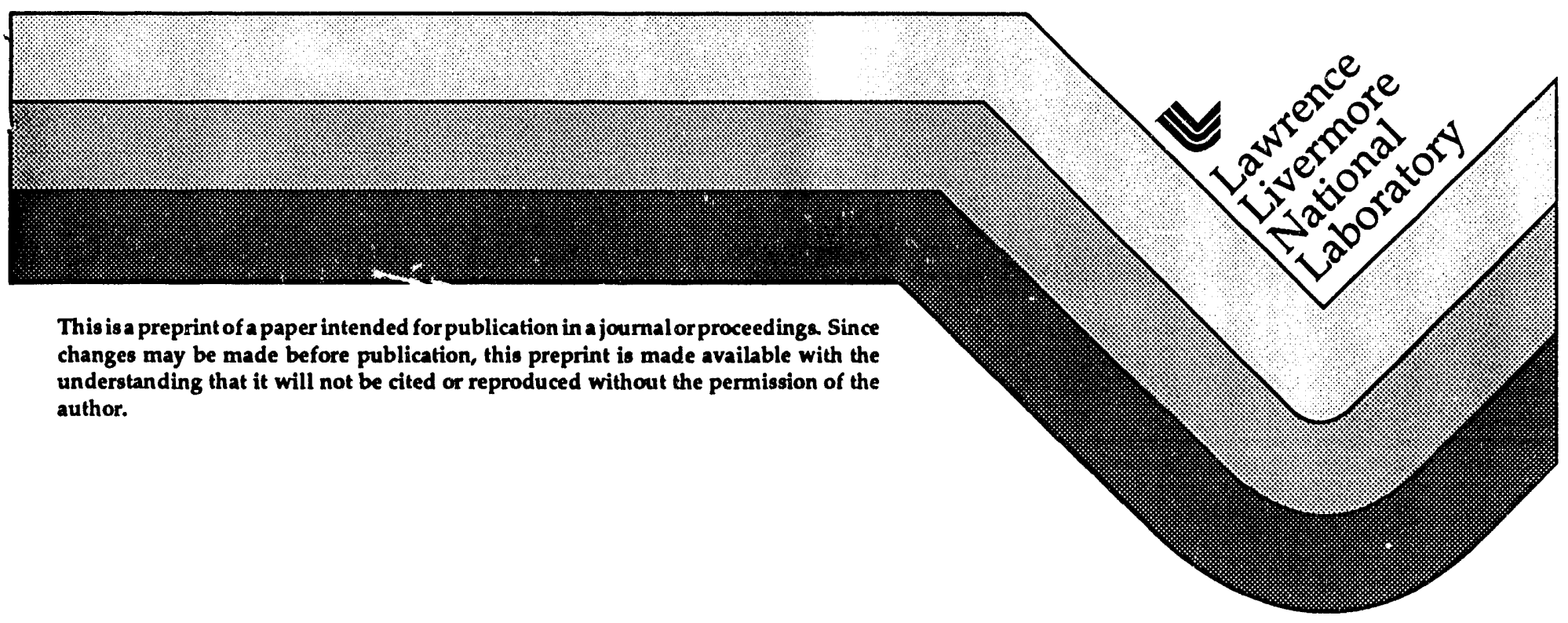




\section{DISCLAIMER}

This document was prepared as an account of work sponsored by an agency of the United States Government. Neither the United States Government nor the Uni versity of California nor any of their employees, makes any warranty, express or implied, or assumes any legal liability or responsibility for the accuracy, completeness, or usefulness of any information, apparat us, product, or process disclosed, or represents that its use would not infringe privately owned rights. Reference herein to any specific commercial products, process, or service by trade name, trademark, manufacturer, or otherwise, does not necessarily constitute or imply its endorsement, recommendation, or favoring by the United States Government or the University of California. The views and opinions of authors expressed herein do not necessarily state or reflect those of the United States Government or the University of California, and shall not be used for advertising or product endorsement purposes. 


\title{
THERMO-HYDRAULIC ANALYSIS OF THE TPX SUPERCONDUCTING TF MAGNETS
}

\author{
Robert L. Wong, Jon P. Zbasnik, William V. Hassenzahl \\ Lawrence Livermore National Laboratory, University of California \\ P.O.Box 808, L-643, Livermore, CA $94551-9900$
}

\begin{abstract}
The superconducting magnets in Fusion Reactors are subjected to pulsed, nuclear. and resistive heating. The thermo-hydraulic response of the helium forcedflow cooled conductors to the various hcat sources is critical to magnet design and performance. Recently developed computer codes allow accurate modeling of conductor response and have been used as an aid to design of the toroidal field (TF) magnets for the Tokamak Physics eXperiment (TPX). We present results of design studies that determine the trade-off between double- and single-pancake helium-flow configurations.
\end{abstract}

\section{INTRODUCTION}

The toroidal field (TF) coils for TPX use helium forced-flow cooled, superconducting, cable-in-conduit conductor (CICC) to permit higher current densities and more compact magnets than is possible with conventional water-cooled copper coils. These conductors are formed by enclosing the cabled superconducting wire strands inside a conduit and swaging the conduit to produce a void fraction of 35 to $40 \%$, which serves as the flow path for supercritical helium. The advantage superconducting coils offer is that no Joule heating is generated during design operation. However, to maintain the superconducting characteristics of the $\mathrm{Nb}_{3} \mathrm{Sn}$ [1], the conductor must be operated at temperatures below the current-sharing temperature. If the temperature rises above current sharing, current will flow in the parallel copper paths provided, resulting in Joule heating. The minimum current-sharing temperature occurs at the bore of the TF coil where the magnetic field is maximum, so the maximum conductor temperature at the bore of the magnet is an important design consideration.

\section{TF COIL OPERATIONAL HEATING AND COOLING}

The coils are designed to remain superconducting during operational heating, which consists of eddy current and hysteresis heating from the pulsed operation of the poloidal field (PF) coils, and steadystate nuclear and gamma heating from plasma radiation and eddy-current heating induced by the vertical stabilization control coils.
Table I

Operational Heat Loading of TPX TP Coils

Direct CICC conductor heating from initial PF pulse

Case heating diffusing into conductor from initial PF pulse $350 \mathrm{~kJ}$

Steady-state nuclear heat

$550 \mathrm{~kJ}$

Steady-state eddy-current heat from vertical stabilization

$3 \mathrm{~kW}$

Operational heating of the 16-coil TF set from various sources is listed in Table $I$.

Pulsed PF heating occurs during a 24-s initiation start-up sequence. Some of that heat is deposited directly into the conductor. The PF heat that is deposited into the case diffuses into the conductor over $500 \mathrm{~s}$. The nuclear heating and the eddy-current heating induced by the vertical stabilization coils are steady state heat loads. We estimate that the coil absorbs $5.3-\mathrm{kW}$ of the $8-\mathrm{kW}$ steady-state heat, with the remainder being intercepted by the auxiliary case cooling.

To remove this heat, the CICC conductor is cooled by force-flowing supercritical (single-phase) helium

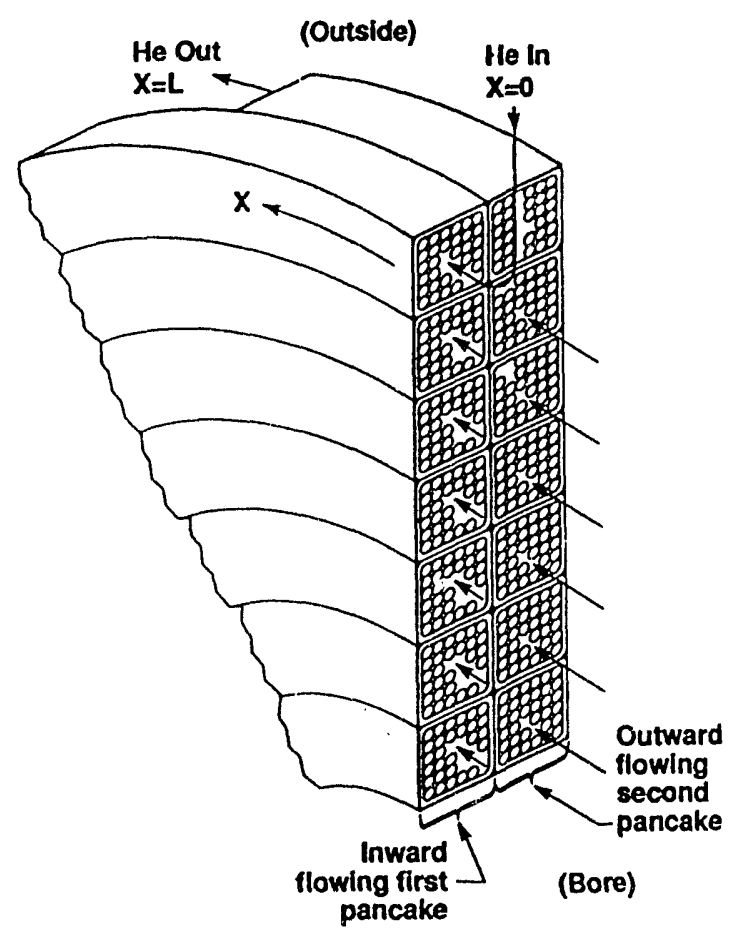

Fig. 1. Double-pancake helium-flow geometry 
through the conductor. In a single-pancake flow configuration, helium flows outward from the bore through one pancake. In a double-pancake flow configuration, helium flows inward toward the bore through the first pancake and outward through the second pancake (Fig. 1). Each conductor is electrically insulated with a G-10 epoxy-impregnated wrap. The single-pancake flow geometry is thermodynamically preferable, since the cold-helium inlet is located at the magnet bore where the field is highest and the currentsharing temperature is lowest. The double-pancake flow configuration is preferred from a reliability viewpoint, since it allows all of the helium connections to be located at the outside of the coil for possible access in case of repair. Twelve pancakes are stacked to form each of the sixteen TF coils. Each pancake has seven turns.

\section{TF COIL OPERATIONAL THERMAL ANALYSIS}

The response of the TF coils to operational heat input was determined using the computer program CICC [2]. This profo: models the CICC conductor by numerically solving the one-dimensional flow continuity, momentum, and energy equations in the helium, along with the axisymmetric heat-conduction equations in the conductor, conduit, and insulation. We compared the maximum temperature at the bore for double- and single-pancake flow configurations.

Figure 1 presents the interpretation of the length coordinate used in program CICC for the doublepancake configuration. The length coordinate, $x$, is the length along the helium flow path, starting at $x=0$ at the helium inlet and ending at $x=L$ at the helium outlet. Each pancake consists of seven turns, each turn being $12.26 \mathrm{~m}$ long. For the double-pancake configuration, $x=0$ is on the outside of the first pancake and $x=L=172 \mathrm{~m}$ is at the outside of the second pancake. The pancake-pancake transition occurs at $\mathrm{x}=\mathrm{L} / 2=86 \mathrm{~m}$ at the bore of the coil. A similar length coordinate exists for the single-pancake configuration with $x=0$ at the bore and $x=\mathrm{L}=86 \mathrm{~m}$ at the outside of the pancake. The model includes the pancake-pancake heat conduction in the double-pancake configuration, and the turn-turn conduction in the double- and singlepancake configurations. Table 2 lists the conductor geometry and the helium and conductor parameters.

Figures 2 and 3 are transient plots in the bore (high field) turn, where the current-sharing temperature is minimum. Figure 2 shows the operational heat input and is a superposition of three different types of heat inputs. The initial 24 -second-long high heat pulse is due to pulsed heating induced into the conductor by the initiation ramp of the PF coils. The 500-second-long intermediate-level heat pulse is the diffusion of PFinitiated eddy-current structural heating into the coil, plus the steady-state nuclear and vertical stabilizer
Table II

CICC Conductor Geometry and Problem Input Parameters
Number of coils

Number of pancakes/coil

Number of turns/pancake

Length per turn

Conductor area

Conductor copper fraction

Heat-transfer perimeter

Conduit area

Conduit heat-transfer perimeter

G-10 insulation thickness

Total inlet helium flow

Inlet temperz ture

Outlet pressure

Operating current
Helium-flow area

Helium-flow hydraulic radius
16

12

$12.26 \mathrm{~m}$

$106 \mathrm{~mm}^{2}$

$0.513 \mathrm{~mm}$

$194 \mathrm{num}^{2}$

0.778

$992 \mathrm{~mm}$

$193 \mathrm{~mm}^{2}$

$80.1 \mathrm{~mm}$

$0.8 \mathrm{~mm}$

$400 \mathrm{~g} / \mathrm{s}$

$5 \mathrm{~K}$

4 atm

$33.5 \mathrm{kA}$ eddy-current heating. The steady-state heating after $500 \mathrm{~s}$ is the nuclear and vertical stabilizer eddy-current heating.

Figure 3 shows the transient-temperature response of the double-pancake configuration to operational heating in Fig. 2. The plotted temperature is at the downstream end of the bore turn (inlet turn) of the second pancake. The temperatures of both the conductor and the outer edge of the G-10 insulation are shown in the figure. The conductor and helium are at the same temperature. The outer edge of the G-10 is the interface between turns. The maximum temperature at the bore is $7.0 \mathrm{~K}$ after 1800 seconds of operation, essentially steady-state. The conductor temperature is higher than the interface temperature, indicating that the heat is flowing out of the conductor. This is expected since the temperature plotted is located in the second pancake. Generally the heat flows from the second pancake to the first pancake. This is the undesirable counterflow heat-exchanger effect of the double-pancake configuration that locates the maximum conductor temperature in the bore of the coil where the current sharing temperature is a minimum.

This counterflow heating effect is also evident in Figs. 4, 5, and 6. These figures are plots along the conductor-flow coordinate of the double-pancake configuration after $1800 \mathrm{~s}$ of operation. Figure 4 shows the distribution of steady-state nuclear and vertical stabilizer heating along the entire flow length. This plot shows only the heat deposited directly into the conductor. Not shown is an equal amount of heat deposited into the sheath, which is also in thermal contact with the helium. The sawtooth pattern represents the conductor alternately spiraling into the low and high heating regions of the coil. The increase in heating toward the bore is due the nuclear heating decay through the coil, with a characteristic decay length of $12.4 \mathrm{~cm}$.

Figure 5 shows the net interface heat flow into the conductor vs flow length at $1800 \mathrm{~s}$. This plot gives 
additional insights into the characteristics and the importance of the pancake-pancake and turn-turn heat flow. The net interface heat flow plotted is the algebraic sum of three components: 1 . heat conducted from the adjacent turn in the other pancake; 2 . heat conducted from the inward adjacent turn in the same pancake; and 3. heat conducted from the outward adjacent turn in the same pancake. The bore turns and outside turns have only two contributing components, since there is only one adjacent turn in the same pancake. Heat flow into the conductor at any given location is positive. An indication of the importance of pancake-pancake and turn-turn heat flow can be seen by comparing the magnitude of the conductor heating (Fig. 4) and the net interface heat flow (Fig. 5). Both the heating and the interface heat flow are generally of the same magnitude. However, at the helium inlet, the interface heat flow is two orders of magnitude more than the operational heating at that location and one order of magnitude greater than the maximum heating anywhere in the conductor. The net interface heat flow is high for the first turn because the helium inlet at $5 \mathrm{~K}$ is located there, also because there is no heat flow out to an adjacent inner turn, and both the heat flow from the second pancake and from the outward adjacent turn are positive (into the conductor). The plot of the net interface heat flow masks the importance of the individual components of the interface heat flow. These individual components can be an order of magnitude greater than conductor nuclear and eddycurrent heating. This means that pancake-pancake and turn-turn heat flow, not the exact shape of the conductor nuclear and eddy-current heating profile, dominates the shape of the temperature profile. Calculations with different operational heat-flow profiles, containing the same total power, do show that the conductor temperature is insensitive to heating profile shape.

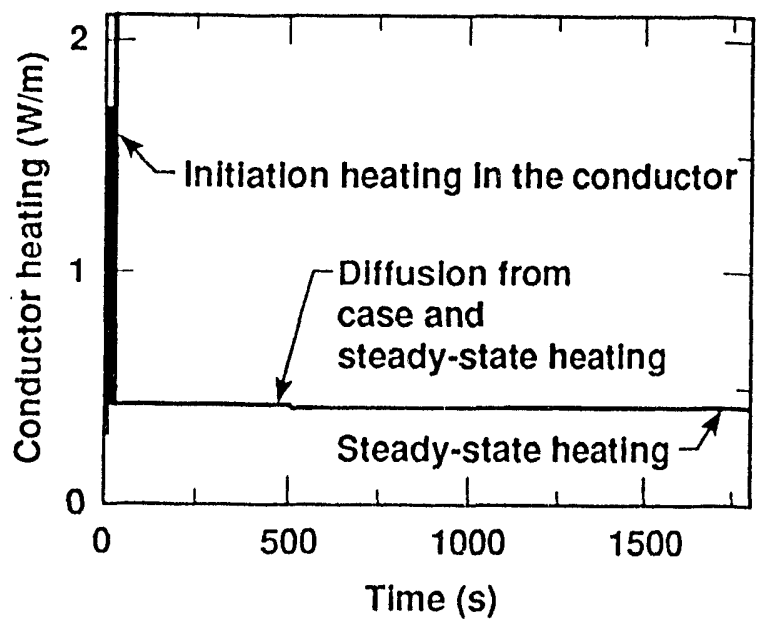

Fig. 2. Transient conductor heating at bore, doublepancake geometry
The temperature profile after $1800 \mathrm{~s}$ is shown in Fig. 6. The peaking of the temperature in the bore, at the transition between the first and second pancakes, is a result of the conductor interface heat flow, particularly the heat flow from the second pancake to the first pancake. Without the inclusion of pancakepancake heat flow, the temperature profile would peak near the helium outlet. The pancake-pancake heat flow is evident from the relative positions of the conductor temperature curve and the interface temperature curve (outside of G-10). In the first pancake the temperature of the interface is above the temperature of the conductor, indicating that the heat flow is into the conductor. In the second pancake the temperature of the interface is below the conductor temperature, indicating that the heat flow is out of the conductor.

The more favorable temperature profile of the single-pancake configuration is shown in Fig. 7. In this configuration the cold-helium inlet turn is located at the bore of the magnet. The conductor temperature continues to increase from the inlet to the outlet at the outside of the magnet. However, any increase in temperature past the first (bore) turn is not important, since the current-sharing temperature increases (due to decreasing field) even more rapidly than does the conductor temperature. Note that outlet temperature is $6.2 \mathrm{~K}$ for the double and single-pancake designs, because each flow path for the double-pancake design has double the heating and double the flow of the single-pancake design. The turn length is $12.26 \mathrm{~m}$ so the maximum temperature occurs at $x=12.26 \mathrm{~m}$, the downstream end of the inlet turn. The maximum steady-state temperature at the bore is $6.0 \mathrm{~K}$. This temperature is $1.0 \mathrm{~K}$ lower than the maximum bore temperature of the double-pancake configuration. The relative positions of the conductor and outside of G-10 plots indicates that there is considerable heat flow from the second turn to the bore turn.

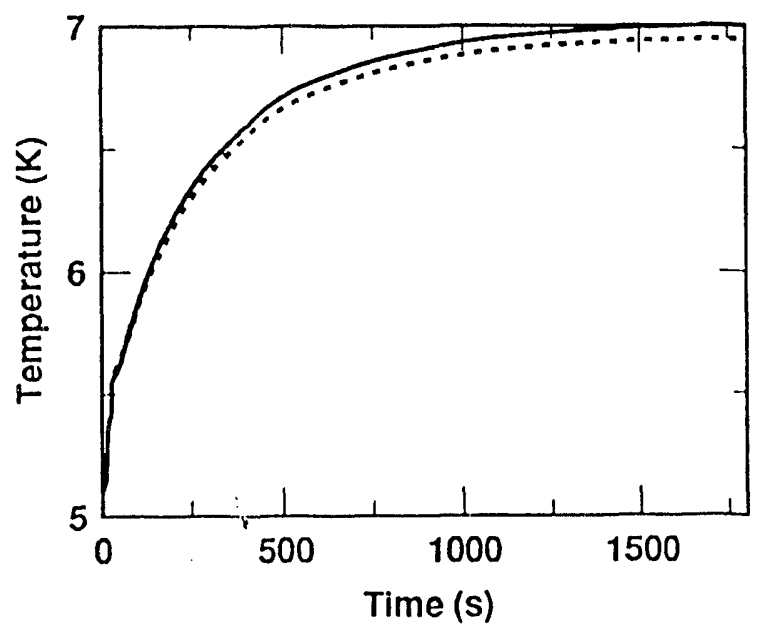

Fig. 3. Maximum transient temperature at bore, double-pancake gerometry 


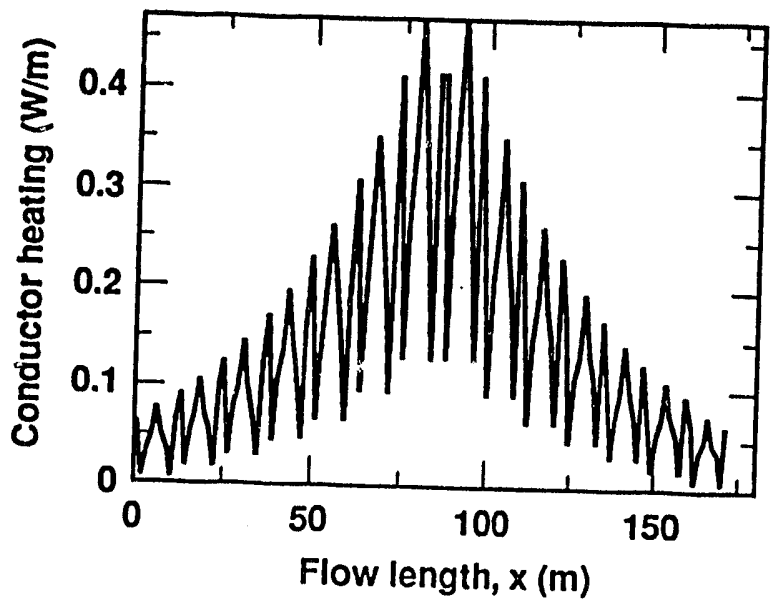

Fig. 4. Steady-state heating profile, doublepancake geometry

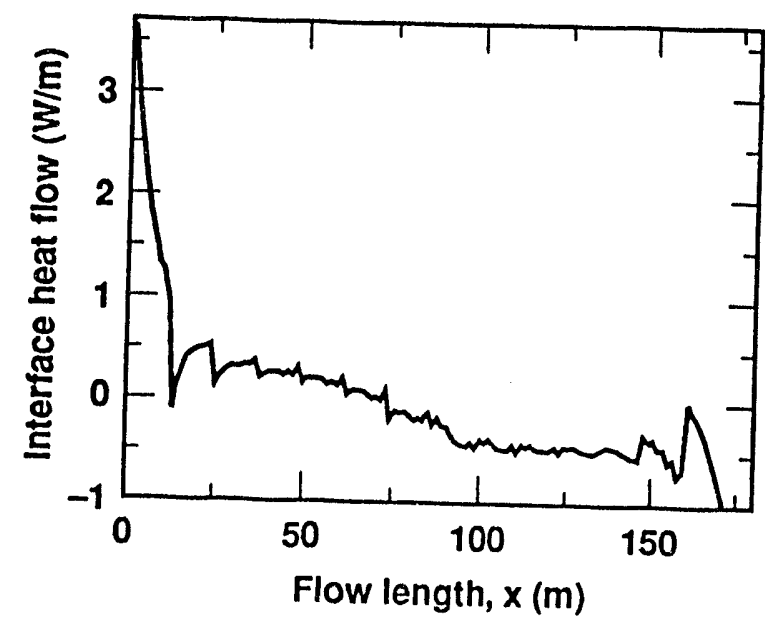

Fig. 5. Net interface heat flow into conductor after 1800-s, double-pancake geometry

\section{CONCLUSIONS}

The analysis shows that the maximum steady-state temperature at the bore for the more reliable doublepancake design is $7.0 \mathrm{~K}$. This is $1.0 \mathrm{~K}$ higher than the $6.0 \mathrm{~K}$ temperature attained at the bore of the singlepancake design. With $400 \mathrm{~g} / \mathrm{s}$ of total helium flow; the steady-state pressure drops for the double and singlepancake designs, respectively, are 3.0 and $0.4 \mathrm{~atm}$.

\section{ACKNOWLEDGMENTS}

This work was performed under the auspices of the U.S. Department of Energy by Lawrence Livermore National Laboratory under contract number W-7405ENG-48.

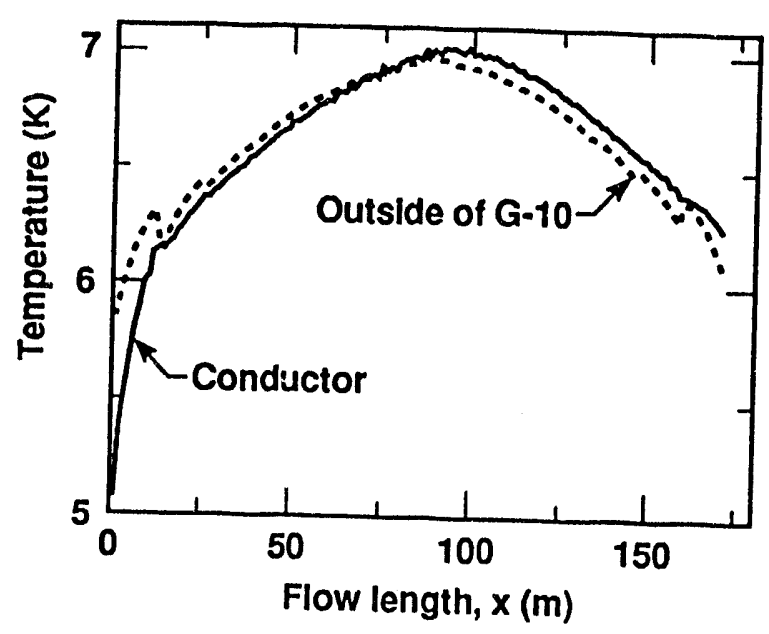

Fig. 6. Temperature profile after $1800-s$, doublepancake geometry

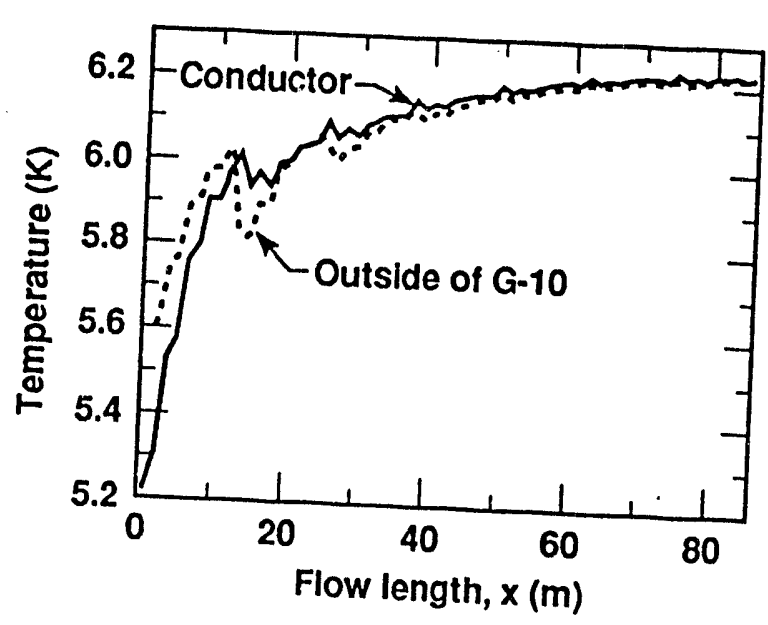

Fig. 7. Temperature profile after 1800 -s, singlepancake geometry

\section{REFERENCES}

[1] M.N. Wilson, "Superconducting Magnets," Clarendon Press, Oxford (1983).

[2] R.L.Wong, "Program CICC, Flow and Heat Transfer in Cable-In-Conduit Conductors--Equations and Verification," Lawrence Livermore National Laboratory, Rept. UCID-21733 (May 22, 1989). 


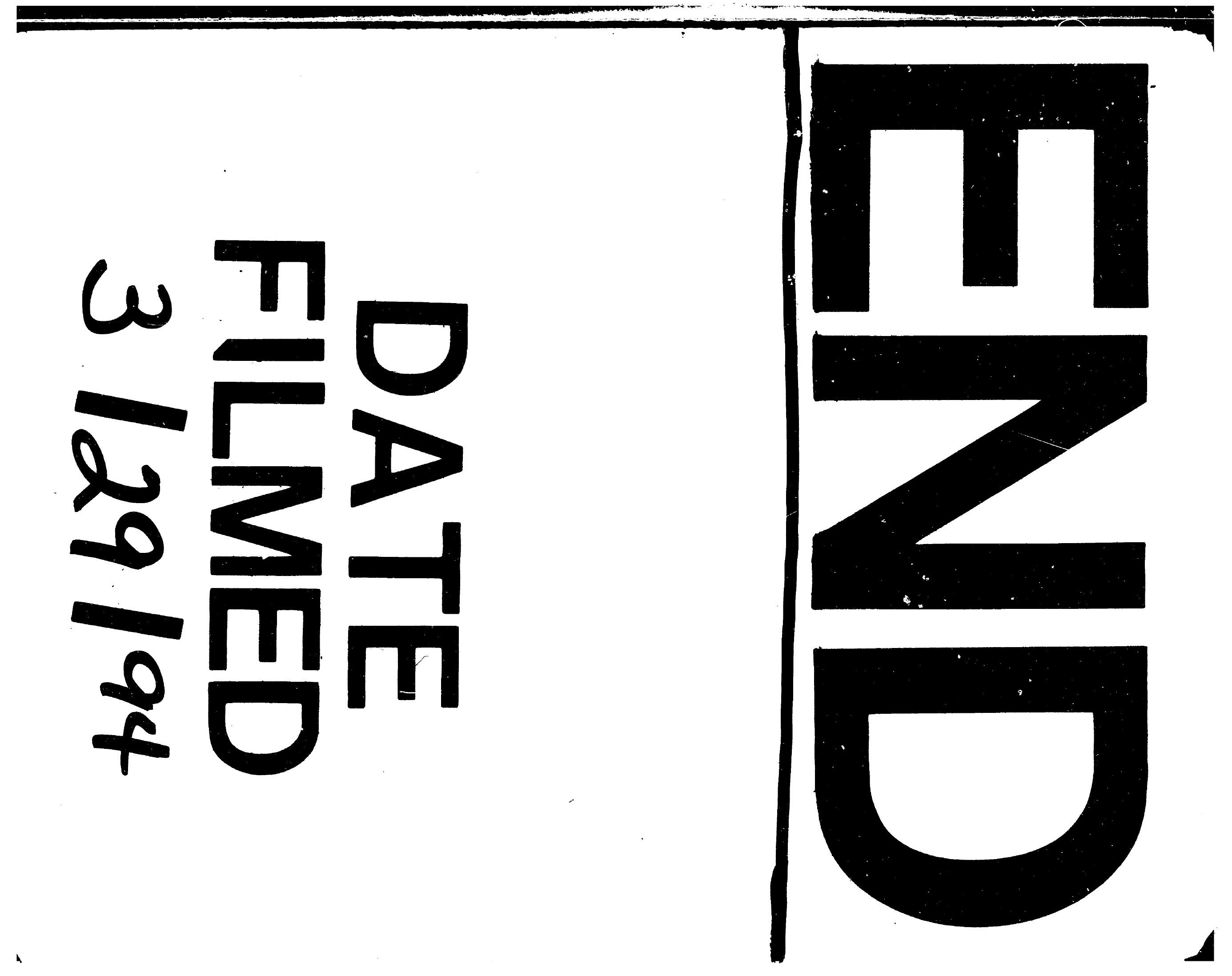



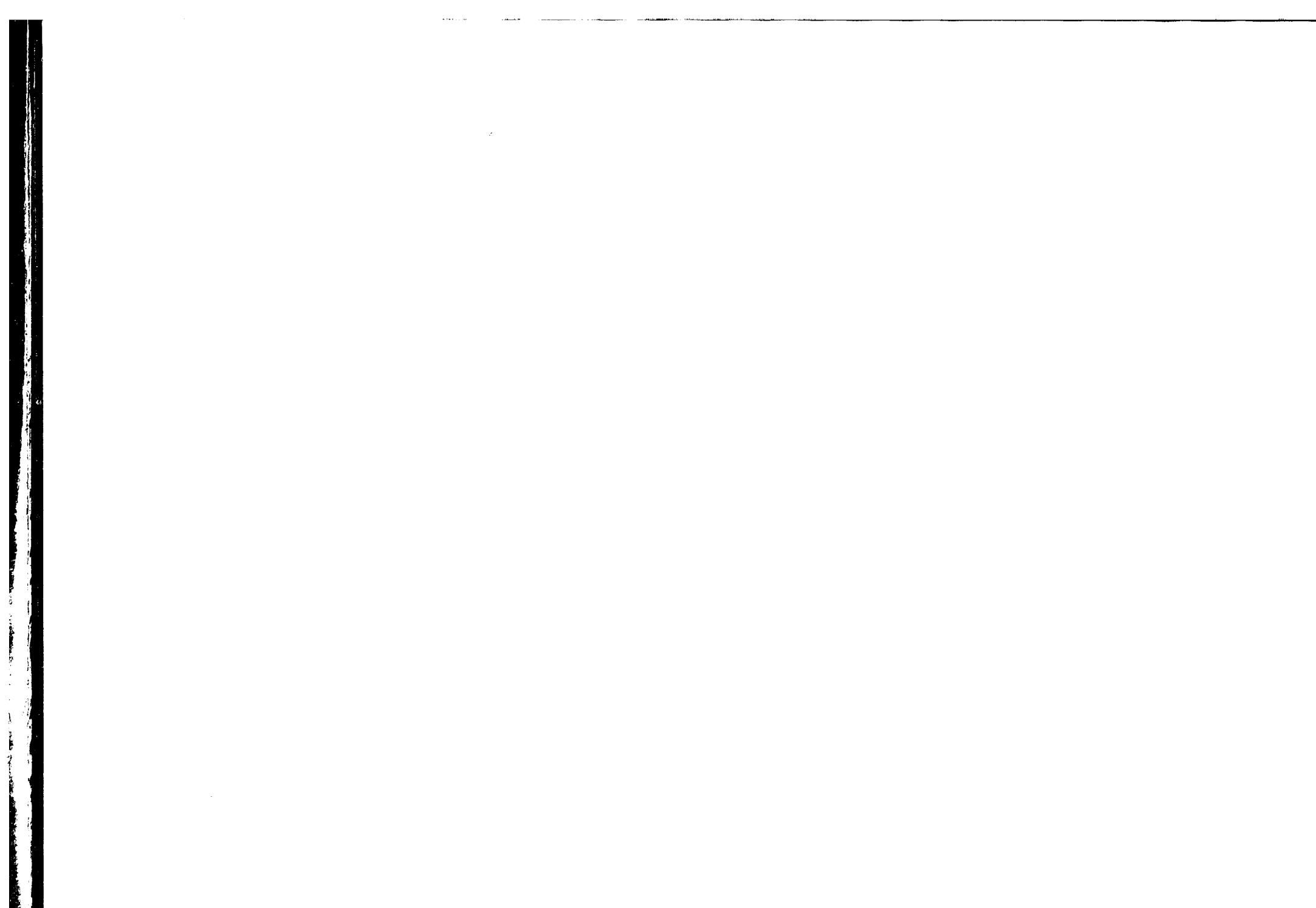